\title{
Opportunities for Isotope Discoveries at FRIB
}

\author{
T. Baumann ${ }^{\mathrm{a}, *}$, M. Hausmann ${ }^{\mathrm{b}}$, B. M. Sherrill ${ }^{\mathrm{a}}$, O. B. Tarasov ${ }^{\mathrm{a}}$ \\ ${ }^{a}$ National Superconducting Cyclotron Laboratory, Michigan State University, 640 S. Shaw \\ Lane, East Lansing, Michigan 48824, USA \\ ${ }^{b}$ Facility for Rare Isotope Beams, Michigan State University, East Lansing, MI 48824, USA
}

\begin{abstract}
Expected production yields of the Facility for Rare Isotope Beams (FRIB) were calculated for a wide range of rare isotopes using the the code $\mathrm{LISE}^{++}$and planned performance parameters [1, 2]. A comparison between isotope discoveries of the last decade and expected particle yields indicates the range of isotopes that can likely be detected at FRIB. This paper will highlight recent isotope discoveries at NSCL's Coupled Cyclotron Facility and deduce how far the limits could be pushed with the Facility for Rare Isotope Beams.

Keywords: Facility for Rare Isotope Beams, isotope discovery, rare isotope beams
\end{abstract}

\section{Introduction}

The discovery of an isotope is not only a prerequisite for the future investigation of its nuclear structure, but it also helps us to delineate the limits of nuclear binding and thus has direct implications for fundamental questions in 5 nuclear physics and nuclear astrophysics. Besides the roughly 300 stable isotopes, there are about 2800 that have been identified so far, but there are still several thousand to be discovered [3, 4,. Isotope discoveries are closely linked to the experimental equipment available, and for rare isotope beams this means that a new generation of accelerator facilities offers opportunities for discovery.

The nuclear drip lines are important study objects because they are sensitive

${ }^{*}$ Corresponding author

Preprint submitted to Nuclear Instruments and Methods in Physics Research BFebruary 23, 2016

(C) 2016. This manuscript version is made available under the Elsevier user license http://www.elsevier.com/open-access/userlicense/1.0/ 


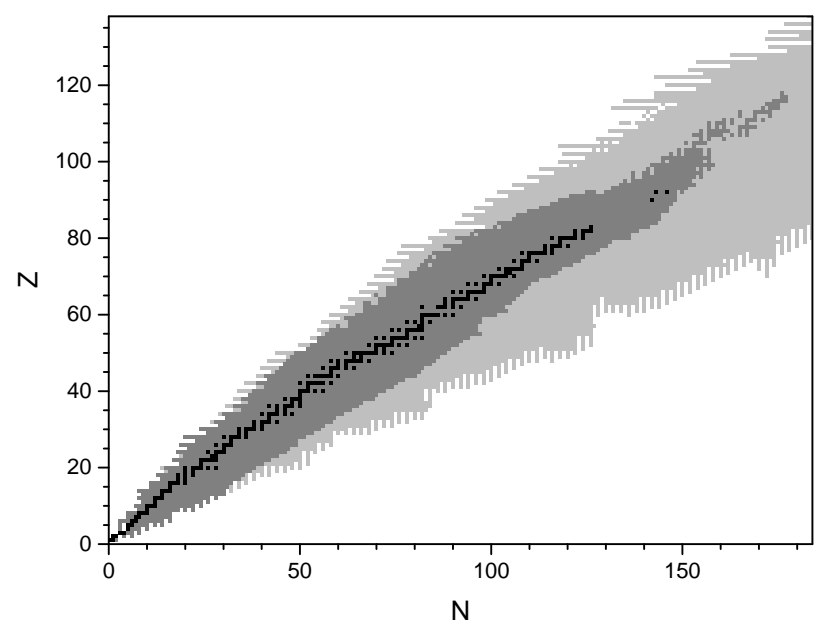

Figure 1: Besides the roughly 300 stable isotopes (black squares), about 2800 radioactive isotopes have been discovered so far (dark grey). Several thousand more are expected to be particle bound (light grey) $3[5$

to the delineation of the limits of atomic nuclei, dependent on aspects of the nuclear force, influenced by nuclear structure changes, and they are central to astrophysical implications.

\section{Methods}

Production yields for the Coupled Cyclotron Facility (CCF) at the National Superconducting Cyclotron Laboratory (NSCL) were calculated with the code LISE $^{++}[1$ version 9.2.68, using the optimum primary beam of a list of 26 available beams ranging from oxygen to uranium and EPAX 2.15 [6] production cross sections. For the ${ }^{238} \mathrm{U}$ beam, projectile fission was also included employing the LISE $^{++}$3EER model [8, 9].

A calculation of all possible rare isotope beams and their expected yields was also done for FRIB in a similar way. In this case, an optimum primary beam out of 47 FRIB primary beams was selected for each isotope. Particle 


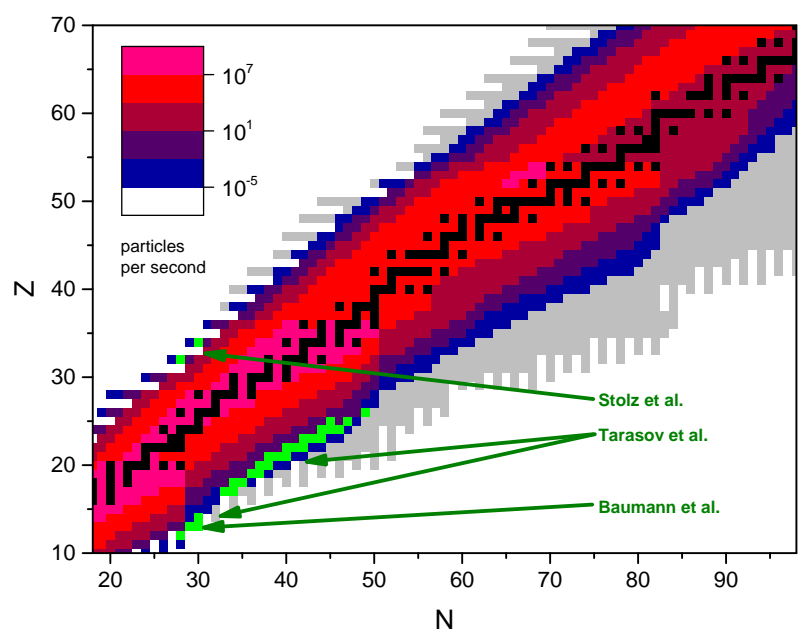

Figure 2: Calculated production yields of NSCL's CCF. Calculations were performed using LISE $^{++}[1$ version 9.2.68. Also indicated (green squares) are NSCL isotope discoveries by Stolz et al. [10, Tarasov et al. [1, 12, 13, and Baumann et al. 14].

yields were calculated using EPAX 3.1 [7] production cross sections, LISE $^{++}$[] version 9.2.68, and the LISE $^{++}$3EER model for in-flight fission 8 .

\section{Results and Discussion}

Figure 2 shows the calculated production yields for NSCL's Coupled Cyclotron Facility on a color scale ranging from $10^{7}$ particles per second down to $10^{-5}$ particles per second. A rate of $10^{-5}$ per second corresponds to about so one particle per day. This figure also highlights new isotope discoveries that were achieved at NSCL during the past decade. These discoveries are based on detecting a small number of particles (3 to 7 ) during typically 7 to 10 day long experiments, and fall into the range of production yield of about one particle per day (taking into account statistical uncertainties). The production cross sections of the most exotic of these isotopes are between $1 \mathrm{pb}$ and $0.01 \mathrm{pb}$ [10, 11, 12, 13, 14 ] 


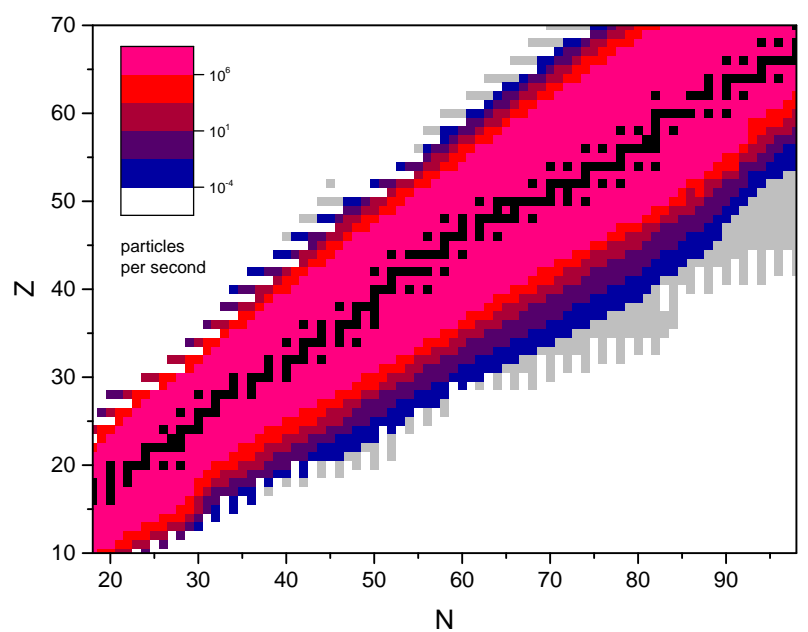

Figure 3: Expected production yields for FRIB. Calculations were done choosing the optimum of 47 available primary beams to create each rare isotope via projectile fragmentation or fission using the code LISE $^{++}[1]$ version 9.2.68.

Comparing the $\mathrm{CCF}$ yields with the discoveries that were possible in the past decade enables us to take a look into the future: What opportunities will there be for isotope discoveries at FRIB?

$40 \quad$ With its high intensity primary beam of $200 \mathrm{MeV} / \mathrm{u}$ and $400 \mathrm{~kW}$, FRIB will make a large number of rare isotopes available for the first time. Besides offering the needed particle yields, the 3-stage fragment separator ARIS [15] will also provide the selectivity that is required to identify these very rare isotopes. The calculated production yields are plotted in Fig. 3 for a selected area of the chart of nuclei.

Looking again at production rates of about one particle per day, or $10^{-5}$ per second, rare isotopes with production cross sections of the order of $10^{-17}$ barn (10 attobarn) should come into reach. This will result in the neutron drip line becoming accessible up to $\mathrm{Fe}$, while at the moment it is only confirmed ${ }_{50}$ experimentally up to oxygen. 


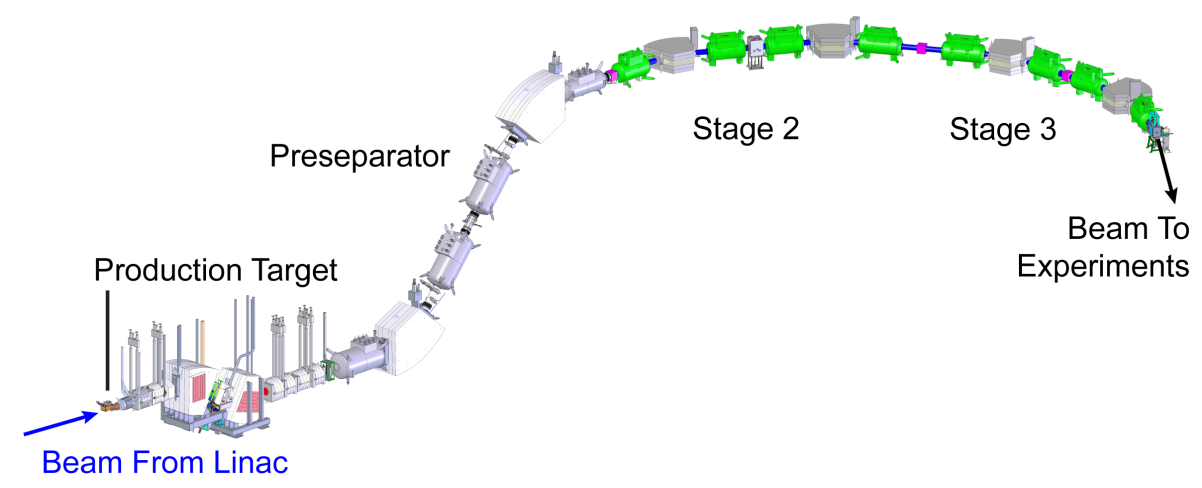

Figure 4: FRIB will employ the Advanced Rare Isotope Separator (ARIS) which has been designed as a 3-stage fragment separator for the efficient collection and purification of rare isotope beams [15.

\section{Conclusion}

FRIB is one of the next generation rare isotope facilities that will ensure a continued stream of rare isotope discoveries together with facilites like the Radioactive Isotope Beam Factory (RIBF) in Japan, the Facility for Antiproton and Ions Research (FAIR) in Germany, and the Système de Production d'Ions RAdioactifs en Ligne de 2e génération (SPIRAL2) in France. Of these facilities only RIBF has come online yet, but in its first 7 years of operation it resulted in the discovery of 69 previously not observed isotopes [16].

When FRIB goes online in 2022 [17] it will further extend the reach to even more exotic nuclei. Besides the new discoveries and expanded delineation of the drip line, FRIB will also enable the study of many key nuclei for astrophysical processes that are beyond the reach of current facilities.

\section{Acknowledgement}

This work was supported by NSF Grant PHY-11-02511. 


\section{References}

[1] O. B. Tarasov, D. Bazin, Nucl. Instr. Meth B 266 (2008) 4657

[2] G. Bollen, M. Hausmann, B. M. Sherrill, O. B. Tarasov, 2011, FRIB Estimated Rates [WWW document], URL

70 https://groups.nscl.msu.edu/frib/rates/fribrates.html (last accessed February 22, 2016)

[3] M. Thoennessen, B. Sherrill, Nature 473 (2011) 25

[4] J. Erler et al., Nature 486 (2012) 509-512

[5] H. Koura et al., Prog. Theor. Phys. 113 (2005) 305

75 [6] K. Sümmerer and B. Blank, Phys. Rev. C 61 (2000) 034607

[7] K. Sümmerer, Phys. Rev. C 86 (2012) 014601

[8] O. B. Tarasov, Tech. Rep. MSUCL1300, NSCL, Michigan State University (2005) [available online], URL http://lise.nscl.msu.edu/7_5/lise++_7_5.pdf (last accessed February 22, 2016)

80 [9] NSCL Rates, 2010 [WWW document], URL https://groups.nscl.msu.edu/frib/rates/nscl_pac35_rates.html (last accessed February 22, 2016)

[10] A. Stolz et al. Phys. Lett. B 627 (2005) 32-37

[11] O. B. Tarasov et al. Phys. Rev. C 75 (2007) 064613

[12] O. B. Tarasov et al. Phys. Rev. Lett. 102 (2009) 142501

[13] O. B. Tarasov et al. Phys. Rev. C 87 (2013) 054612

[14] T. Baumann et al. Nature 449 (2007) 1022-1024

[15] M. Hausmann et al., Nucl. Instr. Meth B 317 (2013) 349 
[16] M. Thoennessen, 2015, Discovery of Nuclides Project [WWW document], URL https://people.nscl.msu.edu/ ${ }^{\sim}$ thoennes/isotopes/ (last accessed February 22, 2016)

[17] FRIB User organization, 2015, [WWW document], URL http://fribusers.org/index.html (last accessed February 22, 2016) 